\title{
Potential immunomodulatory effects of plant lectins in Schistosoma mansoni infection
}

\author{
Eliana A.G. Reis ${ }^{a}$, Daniel A. Athanazio ${ }^{a}$, Benildo Sousa Cavada ${ }^{b}$, Edson Holanda Teixeira ${ }^{c}$, \\ Vicente de Paulo Teixeira Pinto ${ }^{c}$, Theomira M.A. Carmo ${ }^{a}$, Alice Reis ${ }^{a}$, Graziela Trocollia ${ }^{a}$, \\ Julio Croda ${ }^{\mathrm{a}}$, Donald Harn ${ }^{\mathrm{d}}$, Manoel Barral-Netto ${ }^{\mathrm{a}}$, Mitermayer G. Reis ${ }^{\mathrm{a}, *}$ \\ a Fundação Oswaldo Cruz (FIOCRUZ), Bahia, Brazil \\ b Departamento de Bioquímica e Biologia Molecular da Universidade Federal do Ceará, Brazil \\ ${ }^{\mathrm{c}}$ Faculdade de Medicina, UFC, Sobral-CE, Brazil \\ d Department of Immunology and Infectious Diseases, Harvard School of Public Health, 665 Huntington Avenue, Boston, MA 02115, USA
}

\section{A R T I C L E I N F O}

\section{Article history:}

Available online 5 June 2008

\section{Keywords:}

Lectins

Schistosoma mansoni

Cytokines

\begin{abstract}
A B S T R A C T
Lectins are sugar-binding glycoproteins that can stimulate, in a non-antigen-specific fashion, lymphocytes, leading to proliferation and cytokine production. Some lectins are utilized as in vitro mitogenic lymphocyte stimulators and their use as immunomodulators against infectious diseases has been evaluated experimentally. In the experimental murine model, the immune response to schistosomiasis is Th1-like during the initial stage of infection, with a shift towards a Th2-like response after oviposition. We report the response of schistosomiasis patients' $(n=37)$ peripheral blood mononuclear cells (PBMC) to stimulation by lectins, including newly isolated lectins from Brazilian flora, and by Schistosoma mansoni soluble egg antigens (SEA). Cytokine production upon lectin stimulation ex vivo was assessed in PBMC supernatants, collected at 24 and $72 \mathrm{~h}$, by sandwich ELISA to IL-5, IL-10, TNF- $\alpha$ and IFN- $\gamma$. In PBMC from infected patients all but one of the lectins induced a Th2-like cytokine response, characterized by elevated IL-5 production that was higher than that induced by SEA stimulation alone. Our results show that the Th2 environment present during schistosomiasis is not affected and that it may be further stimulated by the presence of lectins.
\end{abstract}

(c) 2008 Elsevier B.V. All rights reserved.

\section{Introduction}

Schistosomiasis is a tropical human disease with 200 million people infected worldwide and almost 600 million at risk (WHO, 2006). The infection, caused by the trematode Schistosoma mansoni, is related to poverty and poor sanitation in contexts where people are exposed to snail-infested water. The most severe human pathology follows granulomatous inflammation of the liver portal tracts leading to hepatic fibrosis, portal hypertension and related complications (Andrade, 2004). Late stage human infection is characterized by a shift from potentially protective Th1-like immune responses to Th2-like responses (Pearce and MacDonald, 2002).

Abbreviations: SEA, S. mansoni soluble egg antigen; Ag, antigen; PBMC, peripheral blood mononuclear cells; Th, T helper cell; IL, interleukin; FCS, fetal calf serum; C-RPMI, complete medium; ELISA, enzyme linked immunosorbent assay.

* Corresponding author at: Fundação Oswaldo Cruz-FIOCRUZ, Rua Waldemar Falcão 121, Candeal, CEP: 40296-710, Salvador, Bahia, Brazil. Tel.: +55 713176 2200; fax: +55 7131762326 .

E-mail address: miter@bahia.fiocmiteruz.br (M.G. Reis).
There is strong evidence from animal models to support a role for Th2 cytokines in the severity of liver granulomatous inflammation and fibrosis (Cheever et al., 2002; Farah et al., 2000; Reiman et al., 2006). The role of Th2 cytokines in human disease is not so clear: peripheral blood mononuclear cell (PBMC) responses with high production tumor necrosis factor $\alpha$ (TNF- $\alpha$ ) and gamma-interferon (IFN- $\gamma$ ) have been associated with the hepatosplenic form (Mwatha et al., 1998) while production of IL-13, IL-4, IL-5 and IL-10 were related to progressive liver disease by others (De Jesus et al., 2004; Montenegro et al., 1999; Alves Oliveira et al., 2006).

Glycan binding proteins (lectins) are a structurally heterogeneous group of reversible carbohydrate binding proteins that are ubiquitous in animals, plants and microorganisms (Van Damme et al., 1998; Gabius and Gabius, 1997; Cavada et al., 2001). Some plant lectins share the ability to act as lymphocyte mitogens and enhance protein-specific immune responses, such as Concanavalin A (ConA) from Jackbean (Canavalia ensiform). ConA has long been used in immunological studies and recently additional plant lectins have been characterized as potent stimulators of immune responses. For instance, $\mathrm{ConBr}$ (from C. brasliensis) activates lymphocytes in vivo (Barbosa et al., 2001) and reduces the size of lesions caused by leish- 
maniasis in BALB/c mice. Although this lectin is a potent stimulator of IFN- $\gamma$ production, the protective mechanism seems to be independent of this cytokine (Barral-Netto et al., 1996). Recently, ConBr and another two lectins were evaluated as Th1-stimulating adjuvants for specific leishmanial antigen immunization and all three proved to be potent stimulators of IFN- $\gamma$. ConBr resulted in lower parasite numbers and a higher inflammatory response in the air pouch model while $\mathrm{KM}+$ (from Arcocarpus integrifolia) was associated with lower parasite counts and reduced lesions after challenge (Teixeira et al., 2006). Thus, although different plant lectins may yield diverse results as in vivo Th1 driving immunostimulants, these observations highlight the potential use of these molecules in conditions in which Th2 polarization are implicated in disease. Importantly, plant lectins with highly conserved DNA sequences and similar binding properties can exhibit distinct biologic effects in different models (Cavada et al., 2001).

The general concept that hepatosplenic schistosomiasis is associated with Th2 response related immunopathology supports the evaluation of Th1-like response stimulants in order to control or revert chronic liver involvement. In this regard, vaccines combining egg antigens and IL-12 resulted in less granuloma formation and reduced tissue fibrosis in BL/6 mice (Sher et al., 1996; Wynn et al., 1995). In contrast, immunization with egg antigen and Freund's adjuvant, in the same mouse strain, resulted in earlier death and larger granulomas in survivors (Rutitzky et al., 2001). In the present work, we report on the effect of lectins, isolated from Brazilian flora, in cytokine production by PBMC of individuals from an endemic area with and without active S. mansoni infection. Our objective was to evaluate the potential immunomodulatory effects of plant lectins from Brazilian flora on S. mansoni infection.

\section{Materials and methods}

\subsection{Subjects}

The individuals included in this study were resident in a schistosomiasis endemic area (Itaquara, Bahia, Brazil). We evaluated 37 individuals aged from 12 to 55 years old with a history of treatment for schistosomiasis. The infection load was determined by quantitative parasitological stool examinations and an estimation of egg number was performed using the modified Kato-Katz method (Katz et al., 1972). Three stool samples from each patient were collected on consecutive days and the geometric mean number of eggs per gram of stool (EPG) was calculated for each individual. All volunteers were informed as to the nature of the research and the samples were collected with the written consent of the subjects, according to a protocol approved by the Human Ethical Committee of the Oswaldo Cruz Foundation. All patients were subsequently treated for schistosomiasis and other parasitic infections.

\subsection{PBMC separation and cytokine assay}

Blood (20 ml) was collected from each individual in heparinized Vacutainer tubes (Sodium heparin BD). PBMC were isolated by centrifugation on a Ficoll-paque gradient from Pharmacia Biotech (Piscataway, NJ, USA) at $400 \times \mathrm{g}, 30 \mathrm{~min}$, at $15^{\circ} \mathrm{C}$. The cells were washed in RPMI 1640 or HBSS (Hank's Balanced Salt Solution) $\mathrm{Ca}^{2+}$ $\mathrm{Mg}^{2+}$ free, and 2-3 $\times 10^{6} / \mathrm{ml}$ were resuspended in RPMI 1640 supplemented with $5 \%$ normal human serum AB, (Sigma-Aldrich), $1 \%$ sodium pyruvate, $100 \mathrm{U} / \mathrm{ml}$ penicillin and $100 \mu \mathrm{g} / \mathrm{ml}$ streptomycin (pen-strep), L-glutamine ( $4 \mathrm{mM}),(\mathrm{GIBCO}$, Grand Island, NY) with or without $10 \mu \mathrm{g} / \mathrm{ml}$ of SEA, $5 \mu \mathrm{g} / \mathrm{ml}$ of each lectins, in 24 well/plates (Costar). The cells were incubated at $37{ }^{\circ} \mathrm{C}$ in $5 \% \mathrm{CO}_{2}$. The super- natants were collected at $24 \mathrm{~h}$ for IL-10 and TNF- $\alpha$, and at $72 \mathrm{~h}$ for IL-5 and IFN- $\gamma$ cytokine production.

\subsection{Antigens and lectins}

Soluble egg antigen (SEA) from S. mansoni was prepared as described elsewhere (Harn et al., 1989). The lectins employed here were derived from the seeds of Brazilian plants belonging to the Diocleinae subtribe (like ConA) and including three genera not used as a food source, Canavalia, Cratylia and Dioclea, as previously described (Dam et al., 1998). Each lectin was isolated according to previously reported procedures (as indicated below). All these lectins have a very close structural identity and share primary carbohydrate recognition specificity for D-mannose and D-glucose and stimulate human lymphocytes in vitro. ConA was used as a control for lymphocyte activation. The plant lectins used in this study were: Cfl from Cratylia floribunda (Oliveira et al., 1991), ConBr from C. braziliensis (Moreira and Cavada, 1984), Dgl from D. grandiflora, (Moreira et al., 1996), Dguil from D. guianensis (Vasconselos et al., 1991), Dros from $D$. rostrata (Cavada et al., 1996a), Dviol from $D$. violacea (Moreira et al., 1996), DvirL from $D$. virgata (Cavada et al., 1996b).

\subsection{ELISA assay}

Cytokine levels were measured at 24 and $72 \mathrm{~h}$ in culture supernatants according to the manufacturer's instructions. TNF- $\alpha$ from Duo-set kits (R\&D System); IFN- $\gamma$ from Genzyme (Cambridge, Mass.) and IL-5 and IL-10 were purchased from (PharMingen, San Diego, CA). Briefly, polystyrene 96 well EIA/RIA plates 3590 from Corning Costar Corp. (NY, USA) were coated with specific mAb, blocked, and incubated with culture supernatants and recombinant cytokines in 96 well/plates, washed then incubated with biotinylated anti-human IgG antibodies. Bound antibodies were detected using an avidin-peroxidase system, and the colored product was measured at $450 \mathrm{~nm}$ in a UV Sofmax automated plate reader and each assay was performed in triplicate.

\subsection{Statistical analysis}

All data in this paper are expressed as a mean \pm S.E.M. unless otherwise described. To compare levels of cytokine production in different groups, the Kruskal-Wallis tested was used for nonparametric analysis. Correlation coefficients between variables were determined by Spearmann rank correlation. Differences with a $P<0.05$ were considered significant. The software used in the present work was GraphPad Prism 4.0 (GraphPad Software, San Diego, CA, EUA).

\section{Results}

\subsection{Demographic and parasitilogic characteristics of subjects}

Subjects were initially separated in two groups: positive for $S$. mansoni eggs ( $n=24 ; 65 \%)$ or negative for schistosomal eggs $(n=13$; $35 \%$ ) in their stools. The mean and standard deviation for age in egg positive and negative groups were, respectively, $20.3 \pm 10.4$ and $37.0 \pm 11.1$ years $(P<0.001)$. Males represented $13 / 24(54 \%)$ and $7 / 13$ (54\%) among the egg positive and negative groups, respectively. The mean EPG of feces was $691 \pm 959$ in the egg positive group. Stratification by infection load resulted in the following groups: $<100$ eggs ( $n=6 ; 25 \%), 100-400$ eggs $(n=6 ; 25 \%)$ and $>400$ eggs $(n=12$; $50 \%$ ). Out of 30 patients, where the stools were also evaluated for other parasites, all but one (in the S. mansoni egg positive group) were infected with other intestinal helminthes, the most common 

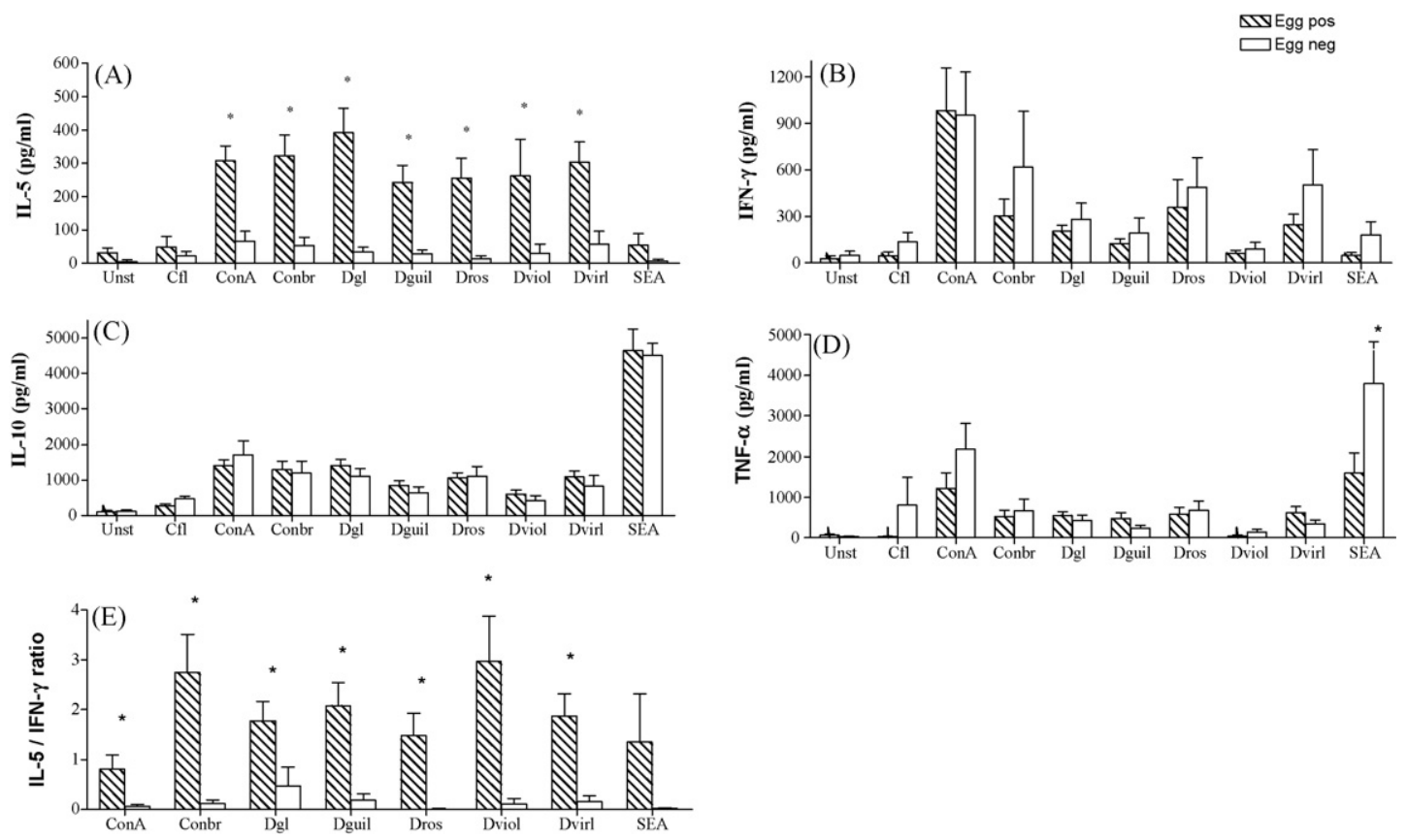

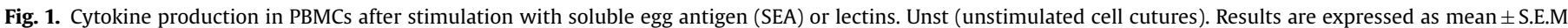

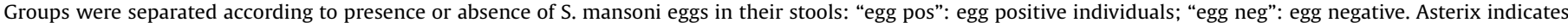
statistical difference between groups.

being Ascaris lumbricoides ( $n=17 ; 57 \%$ of all subjects) and Trichiurus trichiura ( $n=13 ; 43 \%$ of all subjects). A triple infection by $S$. mansoni, A. lumbricoides and T. trichiura was observed in seven patients, the $S$. mansoni egg negative group did not contain individuals infected with both $A$. lumbricoides and $T$. trichiura.

\subsection{Influence of Schistosoma infection on immune response to lectins from Brazilian flora}

As shown in Fig. 1A, there was no difference in IL-5 production between unstimulated PBMC and those stimulated by Cfl or SEA.
Stimulation with Cfl or SEA resulted in a modest IL-5 production, not statistically significant, from unstimulated cultures. In contrast, significant higher cytokine levels for the other seven lectins tested were detected in egg positive patients $(P<0.05)$. While there was no difference upon SEA stimulation between egg positive and negative individuals, seven lectins induced higher IL-5 responses when compared to SEA stimulation: ConA (5.7 $\times, P<0.001), \operatorname{ConBr}(6.0 \times$, $P<0.001), \operatorname{Dgl}(7.3 \times, P<0.001)$, Dguil $(4.5 \times, P<0.001)$, Dros $(4.7 \times$, $P<0.01)$, Dviol $(4.9, P<0.05)$ and $\operatorname{DvirL}(5.6 \times, P<0.001)$.

In contrast to the results of IL-5, there was a trend towards higher IFN- $\gamma$ production in egg negative individuals albeit those differ-
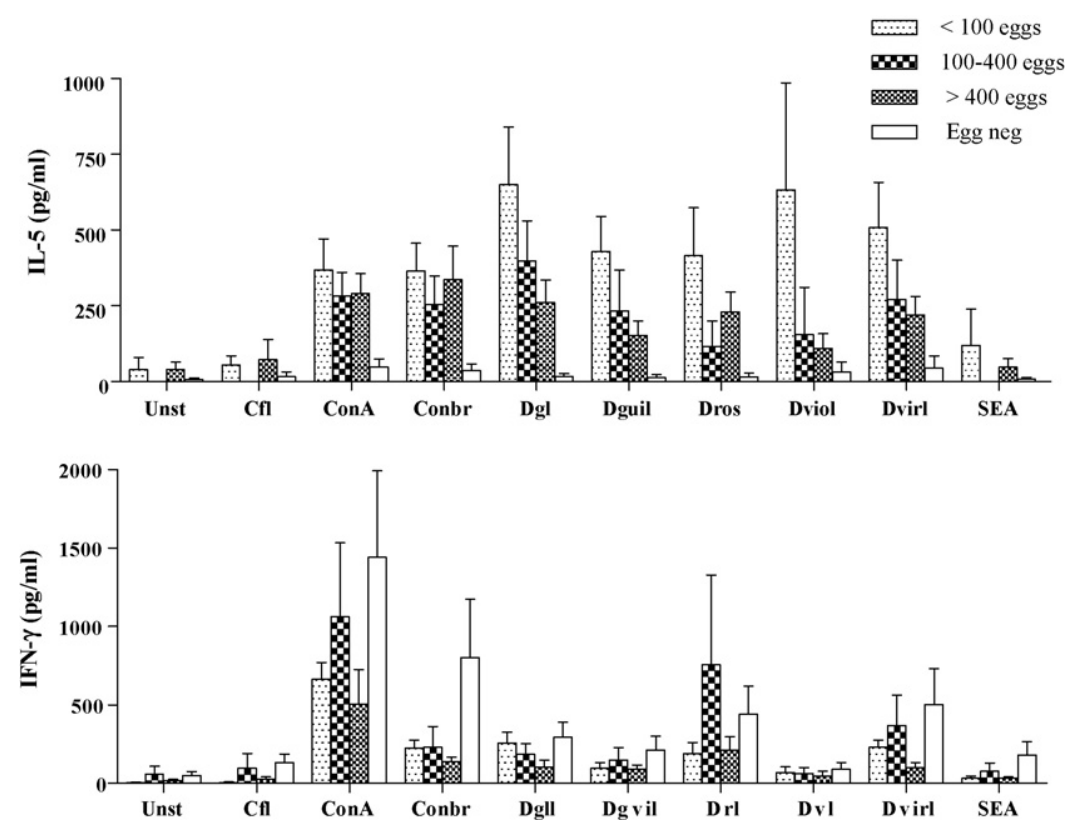

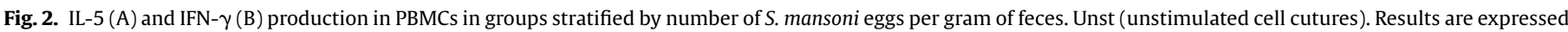
as mean \pm S.E.M. 


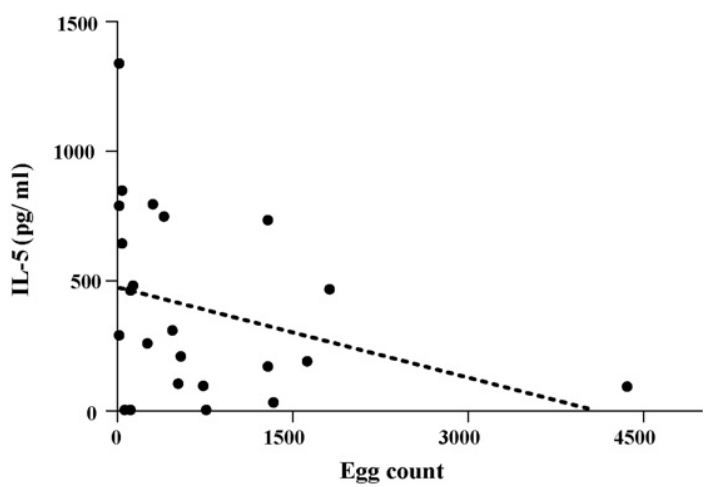

Fig. 3. Negative correlation between IL-5 production and egg count upon Dgl stimulation.

ences between egg-positive and egg-negative individuals were not statistically significant (Fig. 1B). The evaluation of IL-5/ IFN- $\gamma$ ratio confirmed higher values for the seven lectins (ConA, ConBr, Dgl, Dguil, Dros, Dviol and DvirL) among egg positive patients (Fig. 1E).

With regard to IL-10 production, SEA induced higher cytokine production compared to all other stimulants in both egg positive and negative groups. IL-10 production by PBMC did not differ according to the presence of S. mansoni eggs (Fig. 1C).

As shown in Fig. 1D, the highest levels of TNF- $\alpha$ were obtained following SEA stimulation. PBMC from egg positive patients exhibited lower TNF- $\alpha$ production upon SEA stimulation when compared to the egg negative individuals $(P<0.05)$. There were no differences in TNF- $\alpha$ production following stimulation by any of the lectins. When the TNF- $\alpha$ response was compared among the stimulants, SEA induced higher levels compared to all lectins except for ConA, both in egg positive and negative groups.

\subsection{Influence of Schistosoma infection load on IL-5 production}

The relationship between parasite load and IL-5 production was studied. As shown in Fig. 2A, individuals infected with 1-100 eggs exhibited higher IL-5 levels for all stimulants except SEA, when compared to egg negative individuals. In contrast, there was trend in the group infected with $>400$ eggs towards lower IL-5 production and, in these individuals, there was no significant difference upon stimulation by Dguil, Dros, Dviol and SEA among egg positive and negative individuals. As shown in Fig. 2B, no particular trend or significant difference was observed regarding IFN- $\gamma$ production.

All stimulants exhibited a trend towards a negative correlation between infection load and IL-5 production. The strongest (and only significant) negative trend correlation was observed with the Dgl lectin (Fig. 3, Spearmann coefficient $=-0.4351, P<0.05$ ).

\subsection{Influence of gender and age on cytokine production}

In the group of individuals with active $S$. mansoni infection, males had a mean egg count similar to females ( $548 \pm 177$ vs. $860 \pm 378)$ and were in the same age range (21.7 \pm 3.7 years vs. $18.5 \pm 1.7$ years). There was a trend among males towards a higher IL-5 production in egg positive individuals. The difference between males and females was significant upon $\operatorname{ConA}(P<0.05)$ and $\mathrm{ConBr}$ $(P<0.05)$ stimulation (Fig. 4A). Conversely, there was a trend among males towards lower IFN- $\gamma$ levels although the difference was not significant for any one lectin (Fig. 4B).

Gender was not relevant in the production of other cytokines, nor was there a significant correlation between age and cytokine production among the different groups and stimuli used. We did not observe any influence of the helminth co-infections in cytokine production upon stimulation albeit that this analysis was limited due to the low number of subgroups and non-infected individuals (data not shown).

\subsection{Influence of other worm infections on IL-5 production}

As all but one individual enrolled in this study exhibited at least one geohelminth infection, we were not able to study a negative control group for all parasites in endemic area. We investigated the potential influence of other helminthiasis by comparing individuals
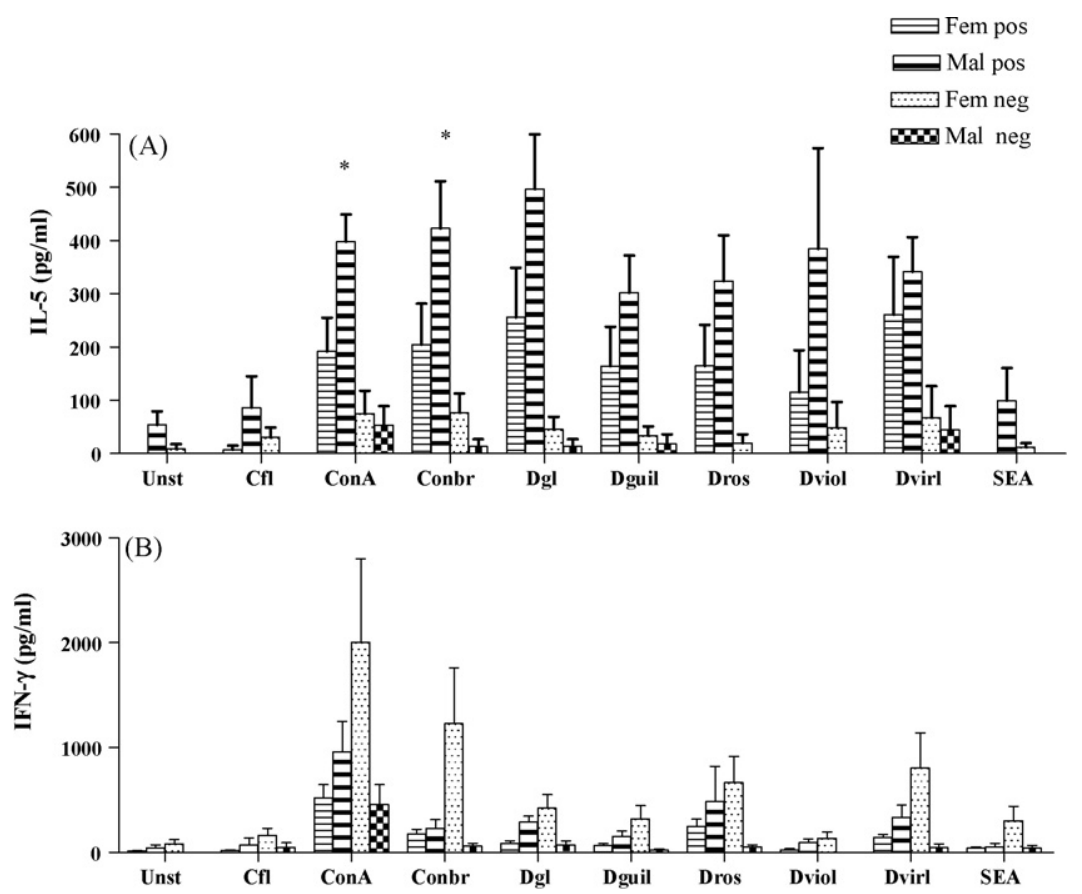

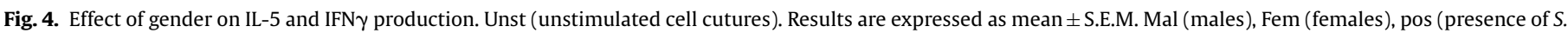
mansoni eggs in their stools), neg (negative for S. mansoni eggs). Asterix indicates statistical difference between groups. 

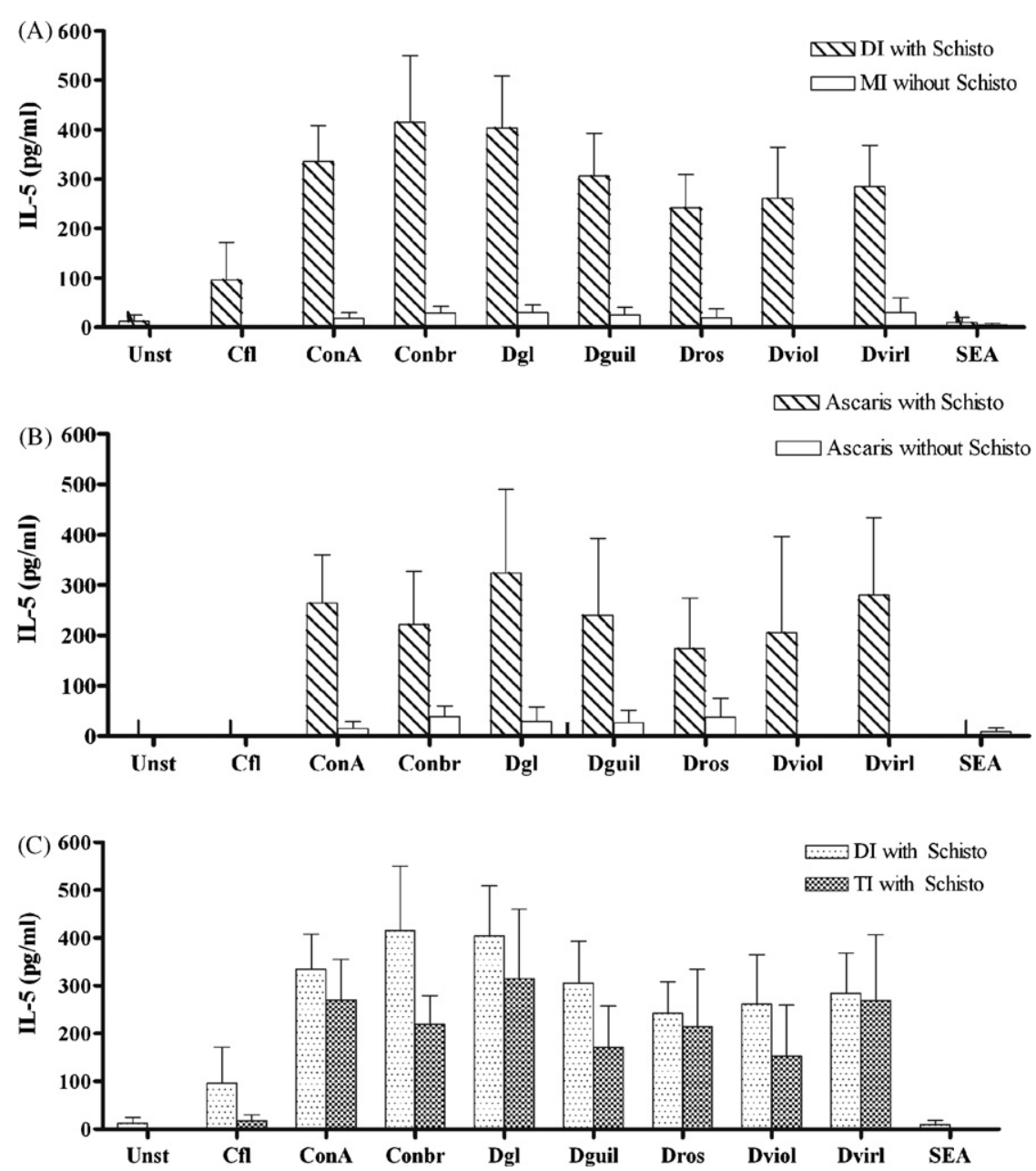

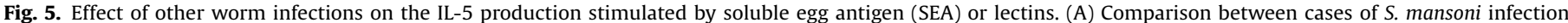

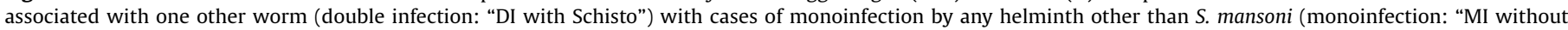

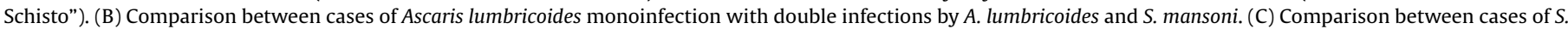
mansoni infection associated with one other worm ("DI with Schisto") with cases of triple infection by S. mansoni and two other helminths ("TI with Schisto").

with any other helminths with and without concurrent S. mansoni infection, individuals with Ascaris lumbricoides infection with and without detectable $S$. mansoni eggs, and individuals with double or triple infections including S. mansoni. No changes in cytokine response were observed when stratifications for other helminthiasis were performed. As seen in Fig. 5, most features described above for IL-5 production were maintained. When considering individuals with one helminth other than S. mansoni, IL-5 production persisted higher in $S$. mansoni infected subjects for all tested stimulants except for SEA (Fig. 5A). When considering individuals with A. lumbricoides infection and no other non-S. mansoni parasite, the trend towards higher IL-5 persisted (even if only seven patients remained as the egg positive group) but significance were reached only for ConBr and Dvirl (Fig. 5B). Moreover, the presence of double or triple infection in S. mansoni infected individuals did not affect cytokine production upon any stimulation (Fig. 5C).

\section{Discussion}

In this study, subjects from an endemic area exhibited a high prevalence of intestinal parasites and, in the case of $S$. mansoni infection, a higher egg count was observed. Individuals with detectable $S$. mansoni eggs were younger, which is in accordance with the established higher prevalence of severe disease in adolescents and young adults. As expected, individuals with active $S$. mansoni infection exhibited lower TNF- $\alpha$ and IFN- $\gamma$ responses to SEA, but the difference for the latter was not significant. Although the difference in IL5 production was not significant among egg positive and negative groups upon SEA stimulation, it was clear that activation by the lectins induced high IL- 5 levels. SEA was a potent stimulator of IL-10 production regardless of an individuals $S$. mansoni egg status while lectins induced lower responses. Previous studies using PBMC from subjects resident in endemic areas from the state of Bahia, Brazil (De Jesus et al., 2004), and other areas (Silveira et al., 2004) confirmed the immunomodulatory properties of SEA on Th1-cytokine downregulation and upregulation of IL-5 and IL-10. As all negative individuals enrolled in our study had a previous history of treated schistosomiasis, the similar patterns of IL-5 and IL-10 production upon SEA stimulation in egg positive and negative groups probably reflects a memory response to SEA. On the other hand, active $S$. mansoni infection appeared to shift the immune response to an unspecific Th2 pattern of cytokine production which could be further induced by lymphocyte stimulants. The active infection includes the exposure to egg and adult worm antigens and their immunomodulatory effects may induce a Th2 polarizing state that is not observed in individuals with past infection.

Strikingly, IL-5 production was clearly higher in egg positive individuals but, in this group, there was a negative correlation between egg counts and IL-5 levels. Recent findings from other 
endemic areas in Brazil report a positive correlation between egg counts and IL-5 levels (Corrêa-Oliveira, manuscript in preparation). There is no clear explanation for this discrepancy. Previously, we showed that an egg sugar, Lacto- $N$-fucopentaose III (LNFPIII), stimulates PBMC regardless of the infection load, although higher IL-10 responses were related to lower egg counts (Velupillai et al., 2000). Egg sugars are targets of lectin binding (Zelck and Becker, 1990; Yoshino et al., 1977) but it is not clear whether lectin activation and the Th2 response induction share similar mechanisms with that of immunomodulation by egg carbohydrates.

In conclusion, we studied the potential role of lectins from Brazilian flora in order to evaluate whether they could polarize PBMC towards a Th1-like cytokine response. Our results indicate that the lectins tested were not able to change the Th2 shift typical of a $S$. mansoni infection. This precludes their use as potential adjuvants or immunomodulatory agents in experimental models of immunization or treatment, in contrast to the beneficial effects seen in experimental leishmaniasis.

\section{Acknowledgements}

This work was supported by Grant AI0546484 from the National Institutes of Health NIH-USA, CNPq 350052/95-6 (NV) and FINEP/PRONEX 4196086200. B.S. Cavada, M. Barral Netto and M.G. Reis are senior investigators of CNPq (Brazil).

\section{References}

Alves Oliveira, L.F., Moreno, E.C., Gazzinelli, G., Martins-Filho, O.A., Silveira, A.M. Gazzinelli, A., Malaquias, L.C., LoVerde, P., Leite, P.M., Correa-Oliveira, R., 2006. Cytokine production associated with periportal fibrosis during chronic schistosomiasis mansoni in humans. Infect. Immun. 74, 1215-1221.

Andrade, Z.A., 2004. Schistosomal hepatopathy. Mem. Inst. Oswaldo Cruz 99, 51-57.

Barbosa, T., Arruda, S., Cavada, B., Grangeiro, T.B., de Freitas, L.A., Barral-Netto, M., 2001. In vivo lymphocyte activation and apoptosis by lectins of the Diocleinae subtribe. Mem. Inst. Oswaldo Cruz 96, 673-678.

Barral-Netto, M., Von Sohsten, R.L., Teixeira, M., dos Santos, W.L., Pompeu, M.L., Moreira, R.A., Oliveira, J.T., Cavada, B.S., Falcoff, E., Barral, A., 1996. In vivo protective effect of the lectin from Canavalia brasiliensis on BALB/c mice infected by Leishmania amazonensis. Acta Trop. 60, 237-250.

Cavada, B.S., Grangeiro, T.B., Ramos, M.V., Cordeiro, E.F., Oliveira, J.T.A., 1996a. Isolation and partial characterization of a lectin from Dioclea rostrata Benth seeds. Revista Brasileira de Fisiologia Vegetal. 8, 31-36.

Cavada, B.S., Ramos, M.V., Cordeiro, E.F., Grangeiro, T.B., Oliveira, J.T.A., De Carvalho, A.F.F.U., Moreira, R.A., 1996b. Purification and partial characterization of a lectin from Dioclea virgata Benth seeds. Revista Brasileira de Fisiologia Vegetal. 8, 37-42.

Cavada, B.S., Barbosa, T., Arruda, S., Grangeiro, T.B., Barral-Netto, M., 2001. Revisiting proteus: do minor changes in lectin structure matter in biological activity? Lessons from and potential biotechnological uses of the Diocleinae subtribe lectins. Curr. Protein Pept. Sci. 2, 123-135.

Cheever, A.W., Lenzi, J.A., Lenzi, H.L., Andrade, Z.A., 2002. Experimental models of Schistosoma mansoni infection. Mem. Inst. Oswaldo Cruz 97, 917-940.

Dam, T.K., Cavada, B.S., Grangeiro, T.B., Santos, C.F., de Sousa, F.A., Oscarson, S., Brewer C.F., 1998. Diocleinae lectins are a group of proteins with conserved binding sites for the core trimannoside of asparagine-linked oligosaccharides and differential specificities for complex carbohydrates. J. Biol. Chem. 273, 12082-12088.

de Jesus, A.R., Magalhaes, A., Miranda, D.G., Miranda, R.G., Araujo, M.I., de Jesus, A.A., Silva, A., Santana, L.B., Pearce, E., Carvalho, E.M., 2004. Association of type 2 cytokines with hepatic fibrosis in human Schistosoma mansoni infection. Infect. Immun. 72, 3391-3397.
Farah, I.O., Mola, P.W., Kariuki, T.M., Nyindo, M., Blanton, R.E., King, C.L., 2000. Repeated exposure induces periportal fibrosis in Schistosoma mansoni-infected baboons: role of TGF-beta and IL-4. J. Immunol. 164, 5337-5343.

Gabius, H.J., Gabius, S., 1997. Glycoscience Status and Perspectives. Chapman and Hall, Weinheim.

Harn, D.A., Danko, K., Quinn, J.J., Stadecker, M.J., 1989. Schistosoma mansoni: the host immune response to egg antigens. I. Partial characterization of cellular and humoral responses to $\mathrm{pI}$ fractions of soluble egg antigens. J. Immunol. 142, 2061-2066.

Katz, N., Chaves, A., Pellegrino, J., 1972. A simple device for quantitative stool thicksmear technique in Schistosomiasis mansoni. Rev. Inst. Med. Trop. Sao Paulo 14, 397-400.

Montenegro, S.M., Miranda, P., Mahanty, S., Abath, F.G., Teixeira, K.M., Coutinho, E.M., Brinkman, J., Goncalves, I., Domingues, L.A., Domingues, A.L., Sher, A., Wynn, T.A., 1999. Cytokine production in acute versus chronic human Schistosomiasis mansoni: the cross-regulatory role of interferon-gamma and interleukin-10 in the responses of peripheral blood mononuclear cells and splenocytes to parasite antigens. J. Infect. Dis. 179, 1502-1514.

Moreira, R.A., Cavada, B.S., 1984. Lectin from Canavalia brasiliensis Mart. Isolation, characterization and behavior during germination. Biol. Plantarum 26, 113-120.

Moreira, R.A., Cordeiro, E.F., Grangeiro, T.B., Ramos, M.V., Martins, J.L., Cavada, B.S. Oliveira, J.T.A., 1996. Isolation and partial characterization of a lectin from Dioclea violacea Benth seeds. Revista Brasileira de Fisiologia Vegetal. 8, 23-29.

Mwatha, J.K., Kimani, G., Kamau, T., Mbugua, G.G., Ouma, J.H., Mumo, J., Fulford, A.J., Jones, F.M., Butterworth, A.E., Roberts, M.B., Dunne, D.W., 1998. High levels of TNF, soluble TNF receptors, soluble ICAM-1, and IFN-gamma, but low levels of IL5 , are associated with hepatosplenic disease in human schistosomiasis mansoni. J. Immunol. 160, 1992-1999.

Oliveira, J.T.A., Cavada, B.S., Moreira, R.A., 1991. Isolation and partial characterization of a lectin from Cratylia floribunda Mart. seeds. Revista Brasileira de Botânica 14, 61-66.

Pearce, E.J., MacDonald, A.S., 2002. The immunobiology of schistosomiasis. Nat. Rev. Immunol. 2, 499-511.

Reiman, R.M., Thompson, R.W., Feng, C.G., Hari, D., Knight, R., Cheever, A.W., Rosenberg, H.F., Wynn, T.A., 2006. Interleukin-5 (IL-5) augments the progression of liver fibrosis by regulating IL-13 activity. Infect. Immun. 74, 1471-1479.

Rutitzky, L.I., Hernandez, H.J., Stadecker, M.J., 2001. Th1-polarizing immunization with egg antigens correlates with severe exacerbation of immunopathology and death in schistosome infection. Proc. Natl. Acad. Sci. U.S.A. 98, 13243-13248.

Sher, A., Jankovic, D., Cheever, A., Wynn, T., 1996. An IL-12-based vaccine approach for preventing immunopathology in schistosomiasis. Ann. NY Acad. Sci. 795 202-207.

Silveira, A.M., Gazzinelli, G., Alves-Oliveira, L.F., Bethony, J., Gazzinelli, A., CarvalhoQueiroz, C., Alvarez, M.C., Lima-Silva, F.C., Prata, A., LoVerde, P.T., Correa-Oliveira, R., 2004. Human schistosomiasis mansoni: intensity of infection differentially affects the production of interleukin-10, interferon-gamma and interleukin-13 by soluble egg antigen or adult worm antigen stimulated cultures. Trans. R. Soc. Trop. Med. Hyg. 98, 514-519.

Teixeira, C.R., Cavassani, K.A., Gomes, R.B., Teixeira, M.J., Roque-Barreira, M.C., Cavada, B.S., da Silva, J.S., Barral, A., Barral-Netto, M., 2006. Potential of KM+ lectin in immunization against Leishmania amazonensis infection. Vaccine 24 3001-3008.

Van Damme, E.J.M., Peumans, W.J., Barre, A., Rougé, P., 1998. Plant lectins: a composite of several distinct families of structurally and evolutionary related proteins with diverse biological roles. Crit. Rev. Plant Sci. 17, 575-692.

Vasconselos, I.M., Cavada, B.S., Moreira, R.A., Oliveira, J.T.A., 1991. Purification and partial characterization of a lectin from the seeds of Dioclea guianensis. J. Food Biochem. 15, 137-154.

Velupillai, P., dos Reis, E.A., dos Reis, M.G., Harn, D.A., 2000. Lewis(x)-containing oligosaccharide attenuates schistosome egg antigen-induced immune depression in human schistosomiasis. Hum. Immunol. 61, 225-232.

WHO, 2006. Schistosomiasis. World Health Organization.

Wynn, T.A. Cheever, A.W. Jankovic, D., Poindexter, R.W., Caspar, P., Lewis, F.A, Sher A., 1995. An IL-12-based vaccination method for preventing fibrosis induced by schistosome infection. Nature 376, 594-596.

Yoshino, T.P., Cheng, T.C., Renwrantz, L.R., 1977. Lectin and human blood group determinants of Schistosoma mansoni: alteration following in vitro transformation of miracidium to mother sporocyst. J. Parasitol. 63, 818-824.

Zelck, U., Becker, W., 1990. Lectin binding to cells of Schistosoma mansoni sporocysts and surrounding Biomphalaria glabrata tissue. J. Invertebr. Pathol. 55, 93-99. 